\title{
Ultrasound mapping system for the surgical management of deep infiltrating endometriosis
}

\author{
Caterina Exacoustos, M.D., ${ }^{a}$ Mario Malzoni, M.D., ${ }^{\mathrm{b}}$ Alessandra Di Giovanni, M.D., ${ }^{\mathrm{b}}$ Lucia Lazzeri, M.D., ${ }^{\mathrm{c}}$

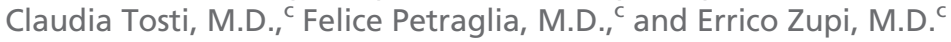 \\ a Department of Biomedicine and Prevention, Obstetrics and Gynecological Clinic, University of Rome "Tor Vergata," Rome; \\ b Advanced Gynecological Endoscopy Center, Malzoni Medical Center, Avellino; and 'Department of Molecular and \\ Developmental Medicine, University of Siena, Siena, Italy
}

Objective: To assess the accuracy of transvaginal sonography (TVS) in defining size and location of deep infiltrating endometriosis (DIE) with laparoscopic/histologic confurmation.

Design: Prospective observational study.

Setting: University hospital.

Patient(s): One hundred four women with suspected DIE on the basis of TVS.

Intervention(s): Patients with DIE underwent TVS evaluation before laparoscopic surgery. An accurate mapping of the extent of the disease was recorded during TVS and at laparoscopy. This new mapping system was developed to assess the extent of endometriosis by measuring the size and depth of the lesions at the various pelvic locations.

Main Outcome Measure(s): Surgical and histologic confirmation of the ultrasonographic data to evaluate the presence and location of DIE and creation of a new mapping methodology for detecting DIE by TVS.

Result(s): Depending on the different location of the lesions, the accuracy of TVS ranged from 76\%-97\%. The lowest sensitivity (59\%) and accuracy (76\%) were obtained for TVS in the diagnosis of vaginal endometriosis, whereas the greatest accuracy (97\%) was shown in detecting bladder lesions and Douglas obliteration.

Conclusion(s): This new ultrasound mapping system is accurate for detecting the extent of DIE and may be useful for preoperative planning and intraoperative management of symptomatic patients with DIE. (Fertil Steril ${ }^{\circledR}$ 2014;102:143-50. (C2014 by American Society for Reproductive Medicine.)

Key Words: Transvaginal ultrasound accuracy, deep infiltrating endometriosis (DIE), laparoscopic surgery

Discuss: You can discuss this article with its authors and with other ASRM members at http://fertstertforum.com/exacoustosc-ultrasound-mapping-system-deep-infiltratingendometriosis/

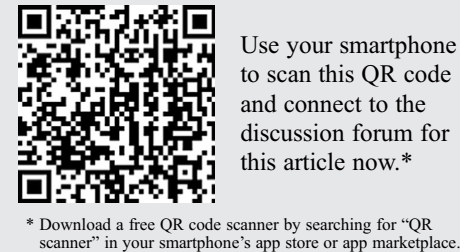
* Download a free $\mathrm{QR}$ code scanner by searching for "QR
scanner" in your smartphone's app store or app marketplace.
$E$ ndometriosis is estimated to affect from 5\%-45\% of reproductive age women. It is associated with significant morbidity and constitutes a major public health concern (1). Deep infiltrating endometriosis (DIE) is defined as an endometri- otic lesion penetrating to a depth of at least $5 \mathrm{~mm} \mathrm{(2)}$ and it affects between $4 \%$ and $37 \%$ of patients with pelvic endometriosis, often causing pelvic pain, dysmenorrhea, dyspareunia, dyschezia, urinary symptoms, and infertility (3). Symptoms caused by DIE are

Received November 26, 2013; revised March 20, 2014; accepted March 21, 2014; published online April 29, 2014.

C.E. has nothing to disclose. M.M. has nothing to disclose. A.D.G. has nothing to disclose. L.L. has nothing to disclose. C.T. has nothing to disclose. F.P. has nothing to disclose. E.Z. has nothing to disclose.

Reprint requests: Caterina Exacoustos, M.D., Department of Biomedicine and Prevention, Obstetrics and Gynecological Clinic, Università degli Studi di Roma "Tor Vergata," Ospedale Generale S. Giovanni Calibita "Fatebenefratelli," Isola Tiberina 1, 00186 Rome, Italy (E-mail: caterinaexacoustos@tiscali.it).

Fertility and Sterility® Vol. 102, No. 1, July 2014 0015-0282/\$36.00

Copyright (02014 Published by Elsevier Inc. on behalf of the American Society for Reproductive Medicine

http://dx.doi.org/10.1016/j.fertnstert.2014.03.043

not specific, often resulting in misdiagnosis or delayed diagnosis $(4,5)$. The pelvic examination may suggest a diagnosis of DIE by the presence of tender nodules and fibrosis in the culde-sac, but it is not very accurate in determining the extent of the disease (6). The current gold standard for a definitive diagnosis of endometriosis remains surgical evaluation with histologic confirmation, although deep retroperitoneal locations could be missed by laparoscopy.

There is a need for a reliable nonsurgical method to diagnose this highly prevalent disease and to determine the extent and location of DIE preoperatively to better plan the 
surgical approach. The level of surgery is strictly related to extent and location of the disease. In certain cases, the treatment requires a multispecialistic surgical team, able to provide the most radical approach and also to avoid the possible medicolegal implications.

To detect DIE extension into the pelvis, use of magnetic resonance imaging (MRI) (7-12) and transvaginal sonography (TVS) (6, 13-18) have been described. The MRI provides accurate information about lesions located on the upper level of the sigmoid, but for pelvic disease TVS is equally accurate and is considered the first and most widely used diagnostic tool $(6,18-20)$. Close communication between the radiologist or sonographer and the clinician is useful to provide the patient with accurate and adequate information regarding the extent of the disease and to plan the best possible management, whether medical, surgical, or both.

The aim of this study was to assess the accuracy of TVS in mapping pelvic DIE by comparing the TVS results with laparoscopic/histologic findings and thus developing a new standardized ultrasound mapping system that accurately records the location, size, and depth of these lesions visualized preoperatively by TVS and subsequently by laparoscopy and histology.

\section{MATERIALS AND METHODS}

This prospective observational study enrolled all patients suspected of having DIE because of their symptoms and who were willing to undergo ultrasonographic evaluation. The DIE was suspected on the basis of clinical history, pelvic examination, and, in particular, symptoms like dysmenorrhea, deep dyspareunia, dyschezia, and dysuria. From January 1, 2011 through June 30, 2012, 104 patients from three endometriosis centers in Italy (Rome, Avellino, and Siena) were included in the study.

The inclusion criteria required a TVS diagnosis of DIE with subsequent laparoscopic evaluation and histologic confirmation within 3 months. Informed consent was obtained from all patients who agreed to take part in the study. Patients without ultrasonographic appearance of DIE did not undergo surgical treatment and were excluded from the study.

A total of 104 women who fulfilled the inclusion criteria were considered in this study, which was Institutional Review Board approved by all three endometriosis centers. Medical, surgical, and obstetric history, as well as signs and symptoms related to endometriosis were recorded for each patient. The level of the patient's discomfort and pain was evaluated by the visual analogue scale system using an 10-point line with the extreme points 0 and 10 corresponding to "no pain" and "maximum pain," respectively.

\section{Mapping System}

A new mapping system, called the Endometriosis SurgicalUltrasonographic System (ESUS) (Fig. 1), was developed to assess the extent of endometriosis by describing accurately the locations of the lesions in the pelvis and by measuring the size and depth of the lesions at the various pelvic sites. This mapping system is based on the anatomical site where DIE could be found and was elaborated by surgeons and a so- nographer together to define exactly each site and to obtain information useful for choosing between surgical or medical treatment. The mapping sheet was filled out first by the sonographer, based on TVS findings, and then, after laparoscopy, by the surgeon. The ESUS was filled by marking the location of pelvic endometriosis divided into four compartments (adnexal, anterior, posterolateral, and Douglas), adding a graphic scheme site by site, and for each lesion the corresponding box option of "yes-no" with relative diameter and depth of infiltration was added.

\section{Ultrasound Examination}

All sonographic examinations were performed by the same examiner (C.E.), with more than 10 years of experience in TVS for DIE. Patients were generally referred for TVS to one center (Rome); otherwise, periodically (1-2 times/mo) the ultrasound examiner visited the other centers (Siena and Avellino).

The ultrasound scan was always performed as a transvaginal examination and, when needed (e.g., in case of rectal DIE) also transrectally with either a GE E8 or E6 (GE Healthcare) ultrasound machine, using a wideband 5- to 9-MHz transducer. No bowel preparation was used before sonography. The examination was performed at any phase of the menstrual cycle regardless of hormonal therapy (HT). All potential locations for DIE in the anterior (bladder) or posterior compartment (vagina, rectovaginal septum, torus and uterosacral ligaments [USL], parametria and ureteral involvement, rectum, and rectosigmoid junction) were examined. The presence of ovarian endometriomas and adnexal adhesions were also recorded. The diagnosis of DIE was made if at least one structure in the anterior or posterior compartment showed the presence of abnormal retroperitoneal hypoechoic linear or nodular thickening with irregular contours and no or few vessels detectable at power Doppler, as per previously described and validated ultrasonographic criteria $(21,22)$.

Anterior compartment (bladder). Patients were requested not to empty their bladder completely before the TVS scan. The slightly filled bladder permits better evaluation of the structure of the walls and the presence of endometriotic nodules. Nodules appear as round-shaped lesions with or without cystic areas and regular/irregular margins of the bladder wall, bulging toward the lumen (Supplemental Fig. 1). Bladder adhesions of the vesicouterine pouch were evaluated by the presence or absence of the "sliding sign" between uterus and bladder. Bladder endometriosis was considered only in case of infiltration of the bladder wall and not in case of only the presence of adhesions.

Posterior compartment. Using TVS and transrectal sonography (TRS) (if needed), an accurate assessment of the vagina, particularly the areas of the posterior and lateral vaginal fornices, the retrocervical area with torus uterinum and USLs, the parametria laterally and the rectovaginal septum, was performed. Rectal sigmoid nodules were visualized as an irregular hypoechoic mass penetrating into the intestinal wall and distorting its normal structure (hypoechoic and thin muscularis propria and hyperechoic submucosa/mucosa) (Fig. 2). For the posterior uterine wall, intestinal nodules 


\section{FIGURE 1}

\begin{tabular}{|c|c|c|}
\hline LOCATION & \multicolumn{2}{|c|}{ LESION } \\
\hline & \multicolumn{2}{|c|}{ POSTERO-LATERAL DIE } \\
\hline \multirow{2}{*}{$\begin{array}{c}\text { UTEROSACRAL LIGAMENTS (USL) and } \\
\text { TORUS }\end{array}$} & \multicolumn{2}{|c|}{ NODULE } \\
\hline & Yes $<1 \mathbf{c m}$ & Yes $1-3 \mathrm{~cm}$ \\
\hline \multirow{10}{*}{ 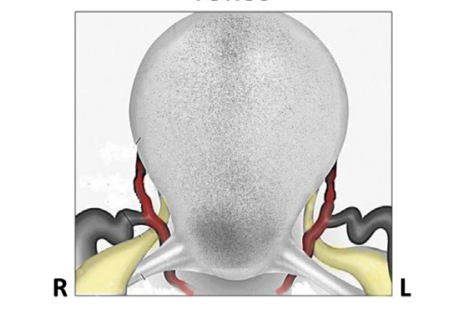 } & \multicolumn{2}{|c|}{ RIGHT USL } \\
\hline & No & Yes \\
\hline & \multicolumn{2}{|c|}{ LEFT USL } \\
\hline & No & Yes \\
\hline & \multicolumn{2}{|c|}{ TORUS } \\
\hline & No & Yes \\
\hline & \multicolumn{2}{|c|}{ RIGHT PARAMETRIUM } \\
\hline & No & Yes \\
\hline & LEFT P & METRIUM \\
\hline & No & Yes \\
\hline \multirow{2}{*}{ PARAMETRIA and URETERS } & \multicolumn{2}{|c|}{ RIGHT URETER } \\
\hline & Comp & Dilatation \\
\hline \multirow{3}{*}{ RECTO-VAGINAL SEPTUM and VAGINA } & & RETER \\
\hline & Comp & Dilatation \\
\hline & & VS \\
\hline & No & Yes \\
\hline & & INA \\
\hline & No & Yes $\ldots \ldots . . . . \mathrm{mm}$ \\
\hline & CRAN & RECTUM \\
\hline & No & Yes \\
\hline & Wall inf & ation grade \\
\hline & Superficial & Full thickness \\
\hline & CAUL & RECTUM \\
\hline & No & Yes \\
\hline CRANI & Wall inf & ation grade \\
\hline & Superficial & Full thickness \\
\hline & DOU & S (D) \\
\hline & DOUGLAS POUCH & LITERATION \\
\hline & Yes partia & Yes complete \\
\hline & ANTE & DR DIE \\
\hline & & DER \\
\hline BLADDER & & ULE \\
\hline & No & $\begin{array}{l}\begin{array}{l}\text { Yes } \\
\text { site : }\end{array} \\
\end{array}$ \\
\hline & Distance from right ureter: & $\ldots \ldots . . . \mathrm{mm}$ \\
\hline & Distance from left ureter: & $\ldots . . . \mathrm{mm}$ \\
\hline & & SIONS \\
\hline & No & Yes \\
\hline & ADNEXAL EN & METRIOSIS \\
\hline & ADN & \\
\hline & ENDOMETR & A (right ovary) \\
\hline ADNEXA & No & Yes ......... mm \\
\hline & ADHESIC & (right ovary) \\
\hline & No & Yes site: \\
\hline & & TUBE \\
\hline & No & Yes $\quad \ldots \ldots . . . . \mathrm{mm}$ \\
\hline & ENDOMETH & MA (left ovary) \\
\hline & No & Yes $\quad \ldots \ldots . . . \mathrm{mm}$ \\
\hline & ADHESIC & (left ovary) \\
\hline $\ln \mathrm{L}$ & No & Yes site: \\
\hline & & TUBE \\
\hline & No & Yes $\quad \ldots \ldots . . . . \mathrm{mm}$ \\
\hline
\end{tabular}

Mapping sheet of the "Endometriosis Surgical-Ultrasonographic System" (ESUS). The ESUS sheet is filled in by marking "yes" or "no" in each box of the chart corresponding to endometriotic locations. The location of the deep infiltrating endometriosis lesion could also be drawn on the anatomical scheme laterally. $L=$ left; $R=$ right.

Exacoustos. DIE ultrasound-surgical mapping system. Fertil Steril 2014. 


\section{FIGURE 2}
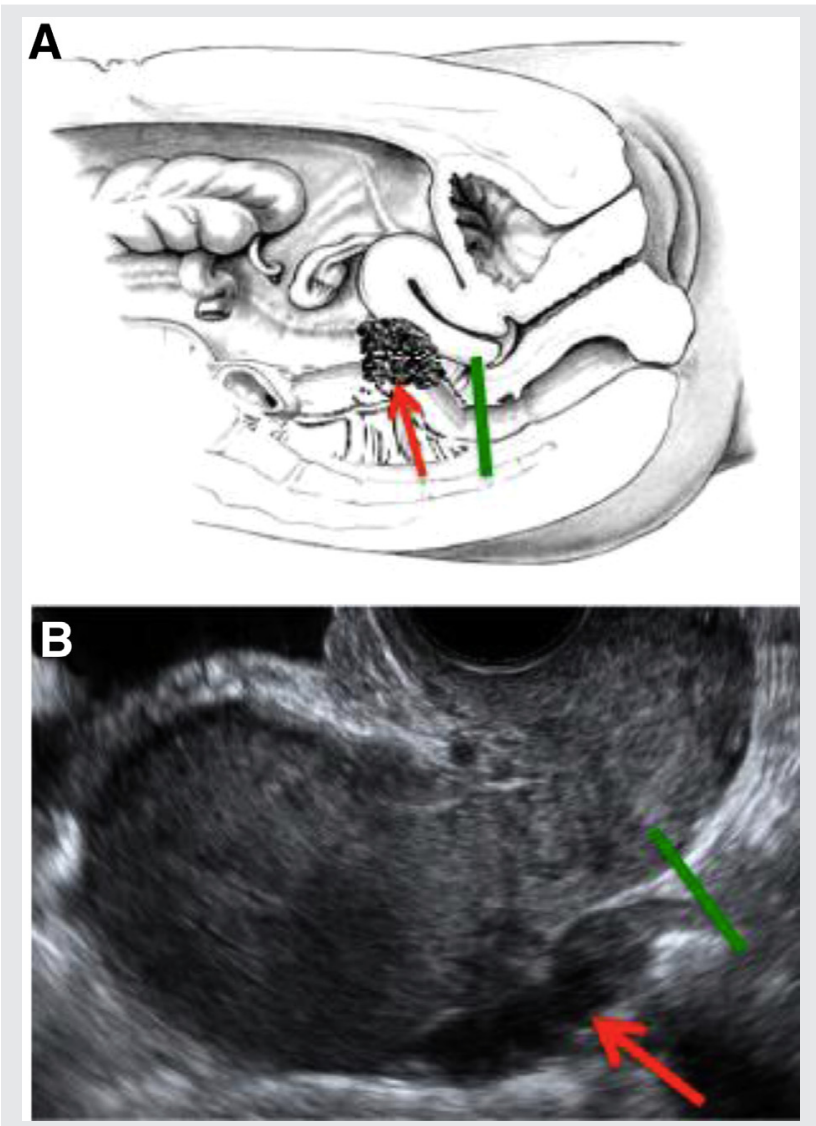

Upper rectal endometriotic nodule. (A) Anatomical schematic showing an upper rectum deep infiltrating endometriosis nodule (red arrow) above the hypothetical horizontal plane passing at the level of the insertion of uterosacral ligaments and dividing low (caudal) and upper (cranial) rectum (green line). (B) Transvaginal ultrasound view showing the uterus in longitudinal section with an hypoechoic retrouterine lesion adherent to the rectosigmoid junction (red arrow).

Exacoustos. DIE ultrasound-surgical mapping system. Fertil Steril 2014.

located below the level of the insertion of the USLs on the cervix were considered low rectal lesions (Supplemental Fig. 2), whereas the ones above this level were considered upper rectal or rectosigmoid junction lesions (Fig. 2).

In case of endometriotic lesions involving the USLs, special attention was paid to the parametrium and to the pelvic ureteral evaluation, particularly in the paracervical area. On the lateral sites where the parametrium is attached to the cervix and at the uterine vessels bifurcation, the parametrial involvement was seen as an infiltrating irregular hypoechogenic tissue that can be medially delimited from the cervical vascular plexuses by using color or power Doppler.

Pelvic ureteral dilation was described by TVS as a tubular anechoic image with or without movements in the parametrial tissue-very similar to a blood vessel but with negative color/power Doppler signs. In case of extrinsic compression without stenosis of the ureter, the TVS diagnosis is more difficult. We identified the distal part of the ureter adjacent to the bladder trigone and followed it laterally to the cervix, and to the pelvic brim, at the level where it crosses the common iliac vessels (23). We suspected an extrinsic compression, even without ureteral dilatation, when a DIE lesion was located close to the ureter. The hypothesis of ureteral involvement suggests a specific and accurate evaluation at the time of surgery, and in these cases transabdominal ultrasound was added to evaluate the renal pelvis.

In all cases, the pain felt by the patient during the examination was recorded, carefully mapping all painful sites ("tenderness-guided" ultrasonography) (21).

\section{Surgery}

Surgery was performed by either of two surgeons with extensive experience in DIE laparoscopic radical resection (M.M. and E.Z.), each with his own surgical team, in two different endoscopic centers, Avellino and Siena. Before surgery the surgeons were familiar with a detailed clinical and ultrasound report for each patient. To match TVS and surgery in mapping DIE lesions, the surgeons were blinded to the sonographic mapping sheet. Surgical diagnosis of endometriosis was based on visualization, whereas measurement was done by using multiples of 5-mm probes. Radical resection of all tissues with endometriotic involvement was performed, followed by histologic confirmation. Bladder or bowel involvement were diagnosed only in case of nodules infiltrating the serosa and muscularis layers, and superficial lesions were considered peritoneal implants that were not visible on TVS (2). Adhesions and all lesions suspected of DIE were removed to adequately assess the extent of disease and obtain its complete resection. When these formations were present, extensive adhesiolysis, excision of ovarian endometriomas, and resection of all visible superficial peritoneal implants and deep lesions were done.

Ureteral resection was done in only a few patients with complete infiltration of the ureteral layers. In most patients, an accurate ureterolysis was performed with a successful dilatation of the distal part. Bladder resection was done by cutting around the lesion and including a visible edge of normal tissue.

In the case of posterior nodules (torus and uterosacral ligaments, parametria, rectovaginal septum, posterior vaginal wall), the rectum was separated from the posterior uterine and/or vaginal wall. In cases of nodules infiltrating the vaginal wall, a full-thickness excision was done.

Lesions of the rectosigmoid were removed by shaving or resection, depending on the size and depth of infiltration of the bowel wall. In the case of lower tract involvement, a stoma was provided, when necessary, to avoid complications. After surgery, the operative report, the surgical ESUS, and the mean operating time of each surgical procedure were recorded.

\section{Statistical Analysis}

For population characteristics all continuous variables are expressed in terms of mean \pm SD. Categorical variables are expressed in terms of frequency and percentage.

Surgical and histologic findings were compared with the preoperative ultrasonographic diagnosis. The prevalence of 
endometriotic lesions at the surgical evaluation was calculated. Sensitivity, specificity, positive and negative predictive values (PVP, PVN), test accuracy, and positive and negative likelihood ratios were calculated with the CATmaker statistical software (Centre for Evidence-Based Medicine) for each site of possible endometriotic infiltration. Our test accuracy is related to the location of DIE in different sites (Fig. 1).

\section{RESULTS}

Laparoscopy revealed DIE in all 104 patients with TVS findings suggestive of the disease, which was further confirmed by histology. Clinical characteristics of the patients and the presence of signs and symptoms are shown in Table 1. Most of our patients had had previous HT (61.5\%) and/or surgery (73\%) for endometriosis. Fifteen of the 104 enrolled patients underwent only medical therapy before surgical treatment, 27 patients had only previous surgeries, 49 patients had surgical and medical therapies, and the other 13 patients did not have any treatment for endometriosis before surgery.

The most common symptoms of our patients with DIE were dysmenorrhea, bowel function symptoms (dyschezia, constipation, diarrhea, rectal bleeding), and dyspareunia. Nulliparity was found in 70 of 104 patients (67.3\%), and 33 of $104(31.7 \%)$ had been infertile for more than 12 months (Table 1).

Sensitivity, specificity, PVP, PVN, positive and negative likelihood ratio, and total accuracy of ultrasound diagnosis compared with surgical findings were calculated for each

\section{TABLE 1}

\begin{tabular}{|c|c|}
\hline Study population characteristics. & \\
\hline $\begin{array}{l}\text { Patient characteristics signs and symptoms } \\
(\mathrm{n}=104)\end{array}$ & $\begin{array}{l}\text { Mean } \pm \text { SD; } \\
\quad \mathrm{n}(\%)\end{array}$ \\
\hline $\begin{array}{l}\text { Age (y) } \\
\text { Parity (n \%) }\end{array}$ & $35.6 \pm 5.7$ \\
\hline 0 & $70(67.3)$ \\
\hline 1 & $20(19.2)$ \\
\hline$\geq 2$ & $14(13.4)$ \\
\hline Previous medical treatment for endometriosis ( $n, \%$ ) & $64(61.5)$ \\
\hline Duration of medical treatment (mo) & $54.1 \pm 61.5$ \\
\hline Previous surgery for endometriosis ( $n, \%)$ & $76(73)$ \\
\hline No. of previous surgical interventions & \\
\hline 0 & $28(26.9)$ \\
\hline $1-2$ & $72(69.2)$ \\
\hline$\geq 3$ & $4(3.8)$ \\
\hline Dysmenorrhea & $96(92.3)$ \\
\hline VAS score ${ }^{a}$ & $8.6 \pm 1.4$ \\
\hline Deep dyspareunia & $65(62.5)$ \\
\hline VAS score ${ }^{a}$ & $7.1 \pm 2.9$ \\
\hline Dyschezia & $67(64.4)$ \\
\hline VAS score ${ }^{a}$ & $7.2 \pm 3.0$ \\
\hline Dysuria & $31(29.8)$ \\
\hline VAS score ${ }^{a}$ & $4.4 \pm 3.3$ \\
\hline Infertility & 33 (31.7) \\
\hline Rectal bleeding & $18(17.3)$ \\
\hline Constipation & $42(40.3)$ \\
\hline Diarrhea & $21(20.1)$ \\
\hline $\begin{array}{l}\text { Note: Signs and symptoms possibly related to the diagnosis of deep } p \\
\text { (infertility: defined as failure to conceive after } 12 \text { months of unprotecte } \\
\text { a Visual analogue scale (VAS) (ranges from } 0-10 \text {, with } 0 \text { corresponding } \\
\text { corresponding to maximum pain). }\end{array}$ & $\begin{array}{l}\text { vic endometriosis. } \\
\text { intercourse). } \\
\text { to no pain and } 10\end{array}$ \\
\hline Exacoustos. DIE ultrasound-surgical mapping system. Fertil Steril 2014. & \\
\hline
\end{tabular}

location and are shown in Table 2. Posterior DIE alone was found at surgery in 91 patients (87.5\%) and anterior DIE alone was found in 1 patient $(0.96 \%)$, whereas the association of posterior and anterior DIE lesions was found in 12 patients (11.54\%). In addition, ovarian endometriosis associated with DIE lesions was detected in 41 patients (39.4\%). Considering the various phases of the menstrual cycle, we did not find statistically significant differences in DIE diagnostic accuracy for different sites of the disease. Comparing the group of patients who had previous surgical treatments (76 patients) with those who never had been treated surgically (28 patients), we did not observe any statistically significant difference in terms of sensitivity, specificity, PVP, PVN, and accuracy. considering the single lesion sites. The group of patients with only medical therapy (15 patients) is too small to be compared with those without medical therapy (13 patients). Bladder endometriosis was found in 8 patients (7.7\%), and it was always TVS-diagnosed (sensitivity 100\%; accuracy $97.1 \%$ ).

In 24 patients showing ureteral involvement, an endometriotic lesion was found around or inside the ureter. In addition, bilateral lesions were found in 5 patients, for a total involvement of 13 on the right ureter and 16 on the left one (Table 2). In all 29 patients, we removed the endometriotic lesion from the ureter and obtained histologic confirmation. Ureteral stents were positioned in 10 patients (9.6\%). A ureteral segmental resection and reanastomosis were performed in three patients $(2.8 \%)$ because of the intrinsic location of the disease. The accuracy of TVS in detecting ureteral involvement was 93.3\% and 91.3\% (right and left ureter, respectively). Intrinsic endometriosis of the ureter is rare, as is the associated dilatation, and in these cases TVS sensitivity is low. An extrinsic disease that may constrict the ureter must be suspected in case of parametrial and USL involvement. The accuracy of TVS in detecting USL endometriotic lesions was $87.3 \%$, whereas for parametrial involvement it was 86.5\% (right parametrium) and 89.4\% (left parametrium). The TVS showed a high PVN and specificity for ureteral location of the disease because it is easier to follow the normal ureter course in the pelvis without endometriotic lesions (Table 2).

We cannot find any statistical differences in the ultrasound diagnostic accuracy in case of small lesions $(<3 \mathrm{~cm})$ compared with larger lesions $(>3 \mathrm{~cm}$ ), in particular revaluating the false-negative cases (lesions not detected by ultrasound and present at surgery). These were not always small lesions but also a large one where the extension and specific site was difficult to evaluate. This was particularly observed for USL, torus, rectal, parametrium, and ureteral lesions. The false-negative rate was the greatest for smaller lesions only in vaginal locations.

Bowel involvement was found in 74 patients: 35 (47.3\%) had nodules of the lower or caudal rectum, and $2(2.7 \%)$ had nodules of the upper or cranial rectum. Intestinal nodules located below the level of the insertion of the uterosacral ligaments on the cervix were considered low or caudal rectal lesions, whereas the ones above this level were considered upper or cranial rectal or rectosigmoid junction lesions. Contiguous lesions of both cranial and caudal rectal segments 


\section{TABLE 2}

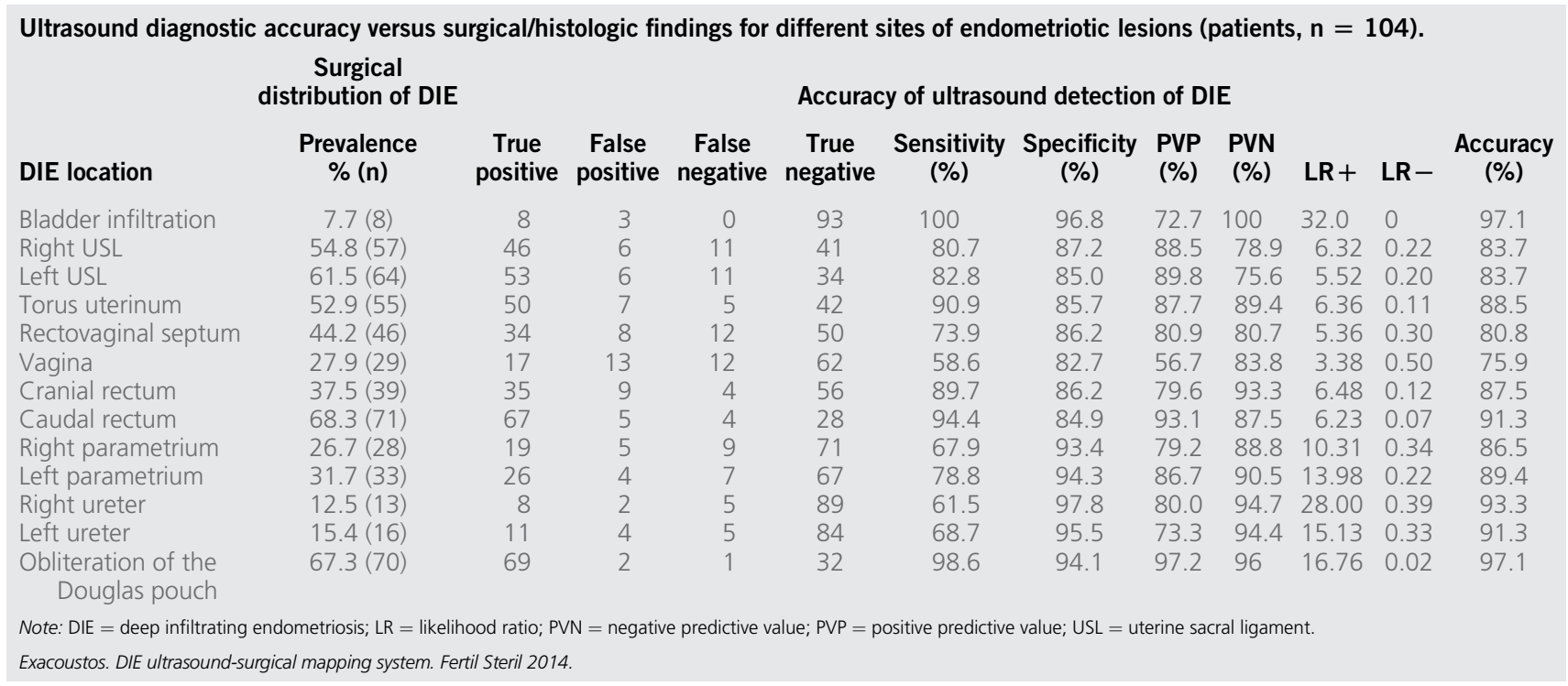

were found in 37 of 74 patients (50\%). The accuracy of the TVS scan of the cranial (87.5\%) and caudal (91.3\%) rectum DIE was high, especially for caudal rectum lesions.

Segmental bowel resection was performed in 25 of 74 patients $(33.7 \%)$. We also observed that all bowel resections presented a maximum diameter of the nodule ranging from 3-6 cm, the mucosa/submucosal layer was infiltrated in 10 patients $(40 \%)$, whereas in the other 15 patients $(60 \%)$ only the muscular layer was involved. To evaluate the diagnostic accuracy of ultrasound in the case of infiltration of the different layers of the bowel wall (transvaginal and transrectal evaluation), we only considered the 25 cases of bowel resection, as histologic specimens were available of the full thickness involvement. The accuracy of ultrasound evaluation in detecting the involvement of the bowel muscular layer was $92 \%$, whereas for mucosal infiltration it was $64 \%$. The lowest sensitivity and accuracy were observed for TVS diagnosis of vaginal endometriosis (sensitivity 58.6\%, accuracy 75.9\%), whereas the greatest accuracy was shown in detecting the Douglas obliteration (97.1\%).

\section{DISCUSSION}

Deep infiltrating endometriosis is a multifocal disease that affects young women of reproductive age and requires skilled, multidisciplinary specialists with a background in the management of the disease. An accurate mapping of endometriotic lesions is essential for optimal therapeutic planning and patient counseling. In our study, a new pelvic disease mapping system (ESUS) based on TVS was developed, resulting in a detailed list of endometriotic lesions. This study demonstrates that the preoperative systematic ultrasonographic evaluation by an expert sonograph operator using ESUS can give an accurate assessment of the presence and location of DIE and inform the surgeon about the actual extent of DIE.

Of the patients included in our study group 73\% had had previous surgery and 62\% had a history of prolonged medical
HT. Even if previous therapy can affect the extent and progression of the disease, a new presurgical DIE mapping is required.

Some investigators (3) describe the possibility of a clinical, not instrumental, diagnosis of DIE, reporting questionable diagnostic accuracy of TVS and MRI, both of which they define as operator dependent. The low accuracy of TVS in detecting vaginal endometriosis, as reported in our study, confirmed that the digital examination could be better than TVS. However, the vaginal examination can easily miss lesions of the cranial rectum and is not able to accurately describe the exact location and dimension of DIE lesions, especially if we consider the lateral and anterior compartments.

The high accuracy for diagnosing bladder endometriosis is concordant with previous published studies, which showed a high level of accuracy in the TVS diagnosis of bladder endometriosis $(24,25)$.

The accuracy of TVS for detecting posterior and lateral DIE lesions (rectum, sigmoid USL, parametria, ureter) is in agreement with previous studies $(6,16,18)$. The rectum, sigmoid, and rectovaginal septum are often considered together, and the Douglas obliteration was described as an indirect sign of DIE $(26)$. Other studies $(17,20)$ reported a lower accuracy compared with our results, probably due to a retrospective analysis of the TVS findings (17) or to the small number of patients (20).

In the treatment of bowel endometriosis, the surgical difficulties are frequently caused by the involvement of the caudal rectum in association with the vagina and the rectovaginal septum, thereby causing a high risk of postoperative complications. In addition, caudal and cranial rectal lesions are often associated with an increased surgical risk and the need for a multidisciplinary surgical team with adequate experience. For this reason, we introduced into our mapping sheet the differentiation between caudal and cranial rectum. When the caudal rectum is involved, its resection can be very challenging and adequate surgical preparation should be entertained, preferably including the 
availability of a colorectal surgeon with experience in laparoscopic surgery.

Although some researchers have investigated the diagnostic value of TVS for detecting rectal endometriosis preoperatively $(6,18-20,25,27)$, data on the estimation of the depth of infiltration are variable. Because the ultrasound scan has low accuracy in diagnosing infiltration of the mucosal layer, TVS does not help surgeons in deciding whether to perform segmental or discoid resection of the lesion. More likely, this decision is dependent on the patient's symptoms, and it is also related to the diameter of the infiltrating tissue and to lumen stenosis. In case of suspected bowel stenosis based on symptoms and on TVS findings, a barium enema could be useful to decide for segmental resection.

The high accuracy of TVS in detecting and describing DIE lesions is similar to or better than that observed in recent studies on MRI (8, 10, 11, 18). All MRI techniques may cause potential discomfort for the patient because of bowel preparation and the use of intravenous contrast medium. They entail higher costs and the need of expert radiologists. For those reasons, it seems that TVS is the best diagnostic tool for mapping DIE.

One limitation of the present study is due to the high prevalence of DIE representing a possible source of bias, especially for descriptive statistical analysis. The patient selection and the setting of our study in three referral centers for endometriosis may explain the high incidence and diagnostic rate of DIE.

The accurate detection of different DIE lesions by TVS, as reported in our study, gives the possibility of surgically managing the disease, when indicated, or alleviating pain with correct medical therapy (whether hormonal or not). This is the first study that describes all the rules that have to be followed by the operator to obtain detailed information about this challenging disease. We know from several published studies that the ultrasonographic diagnosis is accurate and reproducible in detecting DIE and we confirmed these data in our study, but the proposed mapping suggests some new standard steps in performing an ultrasound scan for DIE. The systematic evaluation of the different pelvic sites (vagina, rectovaginal septum, torus uterinum and uterosacral ligaments, parametria and ureter, rectum and rectosigmoid junction) allows an easier and more appropriate diagnostic approach to the disease. The schematic report that appears in the mapping sheet represents an easy-to-follow trail for both ultrasonographer and surgeon.

The Endometriosis Surgical-Ultrasonographic System may be considered a useful tool for preoperative mapping of endometriosis. It has been developed with the specific aim of creating a common language so that physicians who are dedicated to the diagnosis and treatment of patients with severe endometriosis can share clinical data and adequately communicate with one another. It gives clinicians the opportunity to decide on the best surgical approach, to evaluate the potential need to involve other surgical specialists (general surgeon or urologist), to establish a correct, tailored management of the disease, and to properly inform patients of the extent of their disease and the therapeutic options.

This ultrasonographic mapping system, which has been validated by our surgical findings, is useful in the management of DIE, to provide the patient with data that may allow making better informed decisions about managing the disease.

\section{REFERENCES}

1. Giudice LC. Clinical practice. Endometriosis. N Engl J Med 2010;362 2389-98.

2. Koninckx PR, Meuleman C, Demeyere S, Lesaffre E, Cornillie FJ. Suggestive evidence that pelvic endometriosis is a progressive disease, whereas deeply infiltrating endometriosis is associated with pelvic pain. Fertil Steril 1991;55: 759-65.

3. Koninckx PR, Ussia A, Adamyan L, Wattiez A, Donnez J. Deep endometriosis: definition, diagnosis, and treatment. Fertil Steril 2012;98:564-71.

4. Vercellini P, Trespidi L, de Giorgi O, Cortesi I, Parazzini F, Crosignani PG. Endometriosis and pelvic pain: relation to disease stage and localization. Fertil Steril 1996;65:229-304.

5. Nnoaham KE, Hummelshoj L, Webster P, d'Hooghe T, de Cicco Nardone F, de Cicco Nardone C, et al. Impact of endometriosis on quality of life and work productivity: a multicenter study across ten countries. Fertil Steril 2011:96:366-73.

6. Hudelist G, Ballard K, English J, Wright J, Banerjee S, Mastoroudes H, et al. Transvaginal sonography vs. clinical examination in the preoperative diagnosis of deep infiltrating endometriosis. Ultrasound Obstet Gynecol 2011; 37:480-7.

7. Bazot M, Darai E, Hourani R, Thomassin I, Cortez A, Uzan S, et al. Deep pelvic endometriosis: MR imaging for diagnosis and prediction of extension of disease. Radiology 2004;232:379-89.

8. Saba L, Sulcis R, Melis GB, Ibba G, Alcazar JL, Piga M, et al. Diagnostic confidence analysis in the magnetic resonance imaging of ovarian and deep endometriosis: comparison with surgical results. Eur Radiol 2014;24: 335-43.

9. Manganaro L, Vittori G, Vinci V, Fierro F, Tomei A, Lodise P, et al. Beyond laparoscopy: 3-T magnetic resonance imaging in the evaluation of posterior cul-de-sac obliteration. Magn Reson Imaging 2012;30:1432-8.

10. Kruger K, Behrendt K, Niedobitek-Kreuter G, Koltermann K, Ebert AD. Location-dependent value of pelvic MRI in the preoperative diagnosis of endometriosis. Eur J Obstet Gynecol Reprod Biol 2013;169:93-8.

11. Giusti S, Forasassi F, Bastiani L, Cela V, Pluchino N, Ferrari V, et al. Anatomical localization of deep infiltrating endometriosis: 3D MRI reconstructions. Abdom Imaging 2012;37:1110-21.

12. Bazot M, Stivalet A, Daraï E, Coudray C, Thomassin-Naggara I, Poncelet E. Comparison of 3D and 2D FSE T2-weighted MRI in the diagnosis of deep pelvic endometriosis: preliminary results. Clin Radiol 2013;68:47-54.

13. Exacoustos C, Zupi E, Carusotti C, Rinaldo D, Marconi D, Lanzi G, et al. Staging of pelvic endometriosis: role of sonographic appearance in determining extension of disease and modulating surgical approach. J Am Assoc Gynecol Laparosc 2003;10:378-82.

14. Hudelist G, Keckstein J. The use of transvaginal sonography (TVS) for preoperative diagnosis of pelvic endometriosis. Praxis 2009;98:603-7.

15. Hudelist $G$, Oberwinkler KH, Singer CF, Tuttlies F, Rauter G, Ritter O, et al Combination of transvaginal sonography and clinical examination for preoperative diagnosis of pelvic endometriosis. Hum Reprod 2009;24: 1018-24.

16. Savelli L, Manuzzi L, Coe M, Mabrouk M, di Donato N, Venturoli S, et al. Comparison of transvaginal sonography and double-contrast barium enema for diagnosing deep infiltrating endometriosis of the posterior compartment. Ultrasound Obstet Gynecol 2011;38:466-71.

17. Fratelli N, Scioscia M, Bassi E, Musola M, Minelli L, Trivella G. Transvaginal sonography for preoperative assessment of deep endometriosis. J Clin Ultrasound 2013;41:69-75.

18. Vimercati A, Achilarre MT, Scardapane A, Lorusso F, Ceci O, Mangiatordi G, et al. Accuracy of transvaginal sonography and contrast-enhanced magnetic 
resonance-colonography for the presurgical staging of deep infiltrating endometriosis. Ultrasound Obstet Gynecol 2012;40:592-603.

19. Abrao MS, Goncalves MO, Dias JA Jr, Podgaec S, Chamie LP, Blasbalg R. Comparison between clinical examination, transvaginal sonography and magnetic resonance imaging for the diagnosis of deep endometriosis. Hum Reprod 2007;22:3092-7.

20. Holland TK, Cutner A, Saridogan E, Mavrelos D, Pateman K, Jurkovic D. Ultrasound mapping of pelvic endometriosis: does the location and number of lesions affect the diagnostic accuracy? A multicentre diagnostic accuracy study. BMC Womens Health 2013;13:43.

21. Guerriero S, Ajossa S, Gerada M, Virgilio B, Angioni S, Melis GB. Diagnostic value of transvaginal 'tenderness-guided' ultrasonography for the prediction of location of deep endometriosis. Hum Reprod 2008; 23:2452-7.

22. Bazot M, Thomassin I, Hourani R, Cortez A, Darai E. Diagnostic accuracy of transvaginal sonography for deep pelvic endometriosis. Ultrasound Obstet Gynecol 2004;24:180-5.
23. Pateman K, Mavrelos D, Hoo WL, Holland T, Naftalin J, Jurkovic D. Visualization of ureters on standard gynecological transvaginal scan: a feasibility study. Ultrasound Obstet Gynecol 2013;4:696-701.

24. Fedele L, Bianchi S, Raffaelli R, Portuese A. Pre-operative assessment of bladder endometriosis. Hum Reprod 1997;12:2519-22.

25. Bazot M, Malzy P, Cortez A, Roseau G, Amouyal P, Darai E. Accuracy of transvaginal sonography and rectal endoscopic sonography in the diagnosis of deep infiltrating endometriosis. Ultrasound Obstet Gynecol 2007;30: 994-1001.

26. Reid S, Lu C, Casikar I, Reid G, Abbott J, Cario G, et al. Prediction of pouch of Douglas obliteration in women with suspected endometriosis using a new real-time dynamic transvaginal ultrasound technique: the sliding sign. Ultrasound Obstet Gynecol 2013;41:685-91.

27. Koga K, Osuga Y, Yano T, Momoeda M, Yoshino O, Hirota Y, et al. Characteristic images of deeply infiltrating rectosigmoid endometriosis on transvaginal and transrectal ultrasonography. Hum Reprod 2003;18: 1328-33. 


\section{SUPPLEMENTAL FIGURE 1}
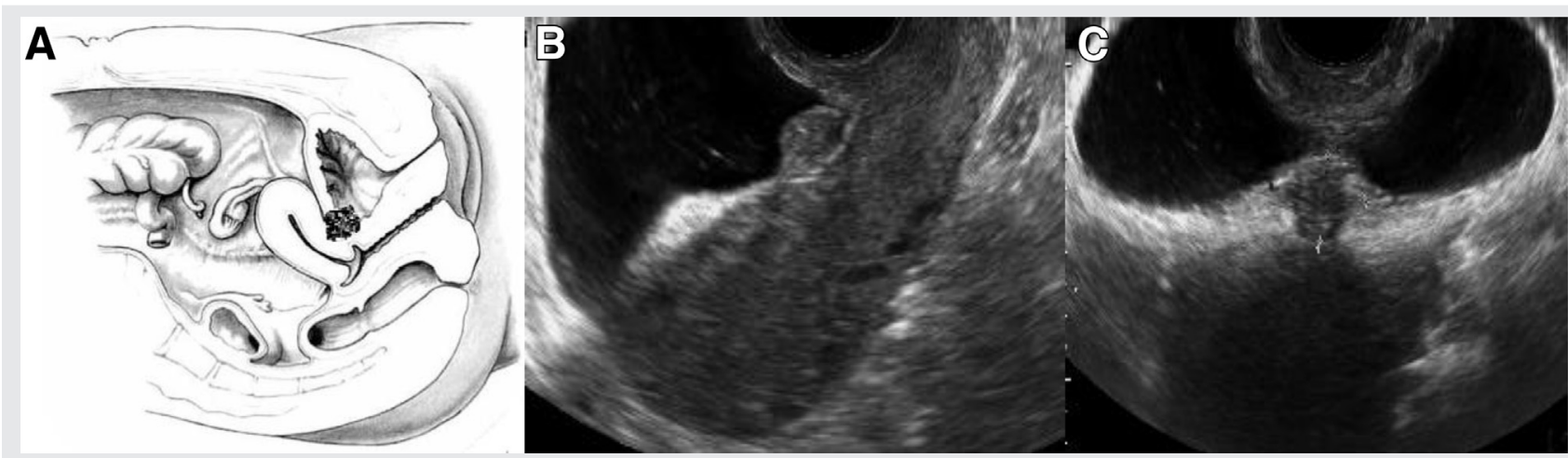

Deep bladder endometriotic nodule. (A) Anatomical schematic; transvaginal ultrasound longitudinal (B) and transverse (C) section. View of the same lesion. Note the round shaped nodule, bulging toward the lumen $(B, C)$ and the adhesions of the vesicouterine pouch and infiltration of bladder wall.

Exacoustos. DIE ultrasound-surgical mapping system. Fertil Steril 2014. 


\section{SUPPLEMENTAL FIGURE 2}
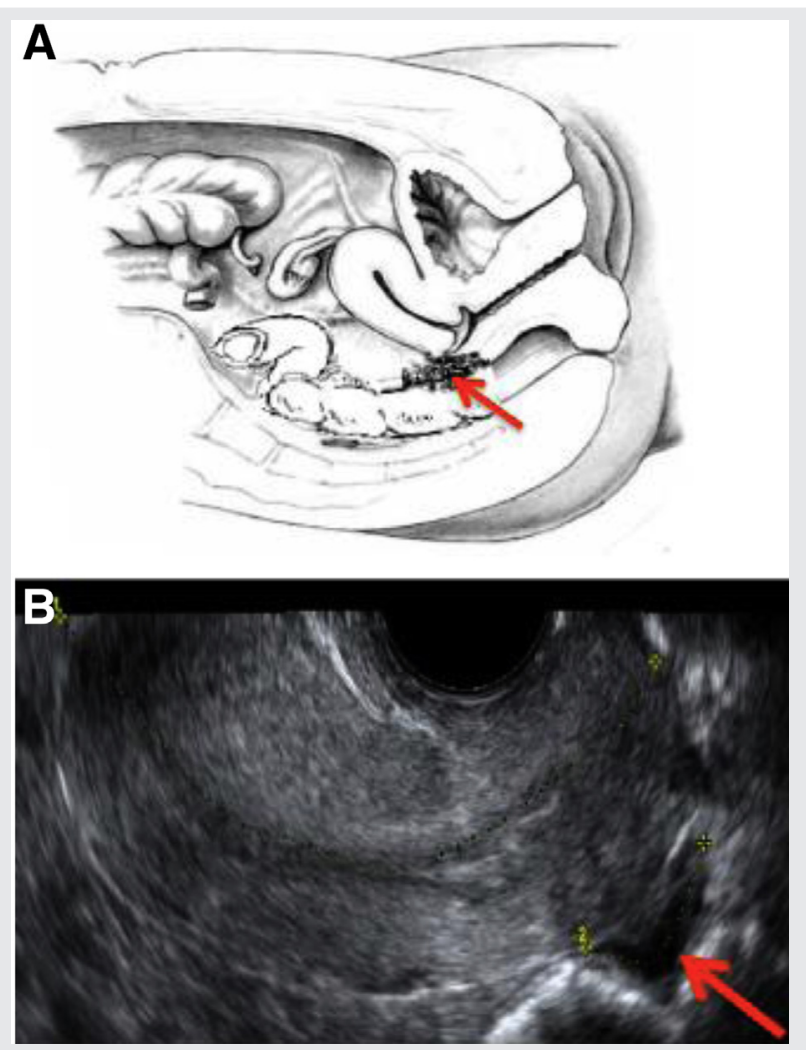

Low rectal endometriotic nodule. (A) Anatomical schematic showing a lower or caudal rectum deep infiltrating endometriosis nodule (red arrow). (B) Transvaginal ultrasound view of uterus in longitudinal section with an hypoechoic retrocervical lesion adherent to the low rectum and the uterine cervix (red arrow).

Exacoustos. DIE ultrasound-surgical mapping system. Fertil Steril 2014. 